\title{
Foundations of Temporal Conceptual Data Models
}

\author{
Alessandro Artale and Enrico Franconi \\ Faculty of Computer Science, Free University of Bozen-Bolzano, Italy \\ \{artale,franconi\}@inf.unibz.it
}

\begin{abstract}
This chapter considers the different temporal constructs appeared in the literature of temporal conceptual models (timestamping and evolution constraints), and it provides a coherent model-theoretic formalisation for them. It then introduces a correct and succinct encoding in a subset of first-order temporal logic, namely $\mathcal{D} \mathcal{L} \mathcal{R}_{\mathcal{U S}}$ - the description logic $\mathcal{D} \mathcal{L} \mathcal{R}$ extended with the temporal operators Since and Until. At the end, results on the complexity of reasoning in temporal conceptual models are presented.
\end{abstract}

\section{Introduction}

Conceptual data models describe an application domain in a declarative and reusable way while constraining the use of the data by understanding what can be drawn from it. A number of conceptual modelling languages has emerged as de facto standards; in particular, we mention entity-relationship (ER) for the relational data model, UML and ODMG for the object-oriented data model, and RDF and OWL for the web ontology languages.

We consider here conceptual modelling languages able to represent dynamic and evolving information in the context of temporal databases 21, 26, 27, 32, 33, 38 40]. We provide in this chapter a mathematical foundation for them by summarising the various efforts appeared in the literature [4, 7, 23, 34, 35]. The main temporal modelling constructs we analyse can be distinguished in two main categories, timestamping and evolution constraints. To support timestamping, the data model should distinguish between temporal and atemporal modelling constructors; this is usually realised by a temporal marking of classes, relationships and attributes that translates into a timestamping mechanism in the corresponding database. A data model supports evolution constraints if it is able to keep track of how the domain elements evolve along time. In particular, status classes describe how elements of classes change their status from being a potential member till they cease forever to be member of the class; transitions deal with the fact that an object may migrate from one class to another one; while generation constraints describe processes that are responsible for the creation/disappearance of objects from classes.

The formalisation is based on a model-theoretic semantics that captures the meaning of both timestamping and evolution constraints. The semantics is obtained as a temporal extension of the model-theoretic semantics associated to 
conceptual models $[14,18]$. The advantage of associating a set-theoretic semantics to a language is not only to clarify the meaning of the language constructors but also to give a semantic definition to relevant modelling notions. In particular, we are able to give a rigorous definition to the notions of: schema satisfiability - when a schema admits a non empty interpretation which guarantees that the constraints expressed by the schema are not contradictory; class and relationships satisfiability - when a class or a relation admits at least an interpretation in which it is not empty; logical implication when a new (temporal) constraint is necessarily true in a schema even if not explicitly mentioned; and finally the special case of logical implication involving subsumption between classes (resp. relationships) - when the interpretation of a class (resp. relationship) is necessarily a subset of the interpretation of another class (resp. relationship).

Building on the provided model-theoretic semantics we provide a correspondence between temporal conceptual models and logical theories expressed in a fragment of first order temporal logic, namely as a Description Logics (DLs) theory. DLs allow for the logical reconstruction and the extension of conceptual models (see [9, 14, 19]). The advantage of using a DL to formalise a conceptual data model lies basically on the fact that complete logical reasoning can be employed using an underlying DL inference engine to verify a conceptual specification, to infer implicit facts and stricter constraints, and to manifest any inconsistencies during the conceptual design phase. In addition, given the high complexity of the modelling task when complex data are involved, there is the demand for more sophisticated and expressive languages than for normal databases. Again, DL research is very active in providing more expressive languages for conceptual modelling (see [13, 14, 17, 18, 18, 19, 24, 31]).

In this context, we consider the temporal description logic $\mathcal{D} \mathcal{L} \mathcal{R}_{\mathcal{U S}}[5]$, a combination of the expressive and decidable description logic $\mathcal{D L R}$ [17] (a description logic with n-ary relationships) with the linear temporal logic with temporal operators Since $(\mathcal{S})$ and Until $(\mathcal{U})$ which can be used in front of both classes and relations. We use $\mathcal{D} \mathcal{L R}_{\mathcal{U S}}$ both to capture the temporal modelling constructors in a succinct way, and to use reasoning techniques to check satisfiability, subsumption and logical implication. The mapping towards DLs presented in this chapter builds on top of a mapping which has been proved correct in 3,4$]$ while complexity results and algorithmic techniques can be found in [1, 5, 11]. Even if full $\mathcal{D} \mathcal{L} \mathcal{R}_{\mathcal{U S}}$ is undecidable we address interesting modelling scenarios where subsets of the full $\mathcal{D} \mathcal{L} \mathcal{R}_{\mathcal{U S}}$ logic is needed and where reasoning becomes a decidable problem.

The chapter is organised as follows. Section 2 describes the temporal constructs that will be the subject of the formalisation. Section 3 shows the modelling requirements that lead us to elaborate the rigorous definition of the framework presented here. Section 4 introduces the model-theoretic semantics and the notions of satisfiability, subsumption and logical implication for temporal conceptual models. The two Sections 5, 6 are the core sections where we describe how timestamping and evolution constraints can be formalised. After presenting the $\mathcal{D} \mathcal{L R}_{\mathcal{U S}}$ logic in Section 7 we proceed with a $\mathcal{D} \mathcal{L} \mathcal{R}_{\mathcal{U S}}$ encoding of the 


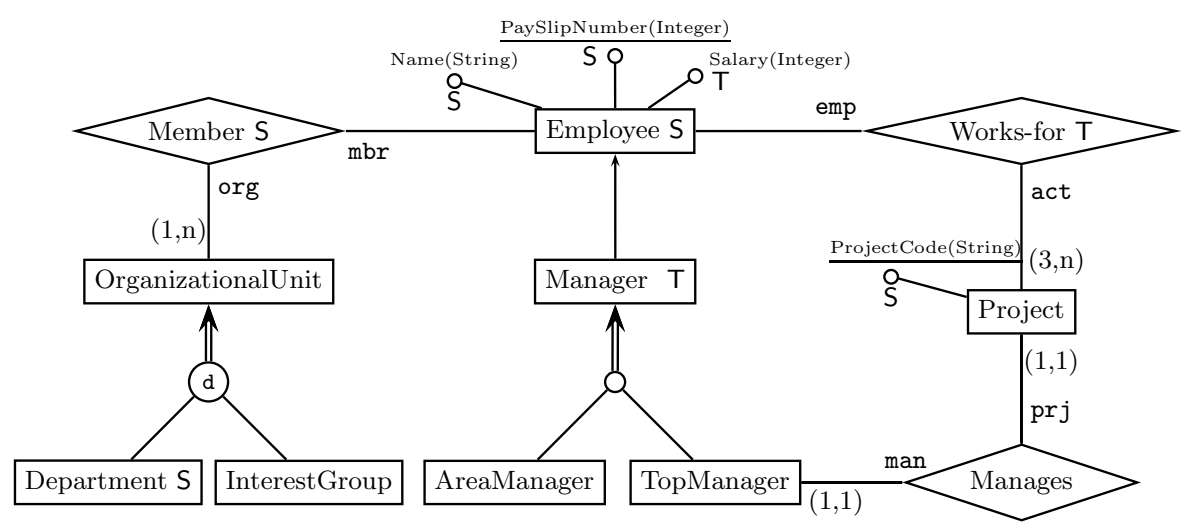

Fig. 1. The Company example

various temporal constructs in Section 8 Section 9 investigates the complexity of reasoning over temporal conceptual models and presents various scenarios where sound, complete and terminating procedures can be used. In Section 10 we state our final remarks.

\section{Temporal Modelling Constructors}

Temporal constructs are usually added to the classical constructs to capture the temporal behaviour of the different components of a conceptual schema. In this chapter we distinguish them in two generic classes: Timestamping and Evolution constructs.

Timestamping. It is concerned with the discrimination at the schema level between those elements of the model that change over time and others that are time invariant. Timestamping applies to classes, relationships and attributes. Data models should allow for both temporal and atemporal modelling constructors. Timestamping for attributes allows keeping how an attribute of a given object changes over time. For example (see Figure 1), the salary of an employee emp-123 has value " $2.5 K \$$ " for the period from $01 / 2004$ to $12 / 2005$, then " $3.0 K$ $\$$ " from $01 / 2006$ to $12 / 2007$, then "3.2K \$" from $01 / 2008$ to $12 / 2009$.

Similarly, temporal periods can characterise an object or relationship instance as a whole rather than through its attributes. Membership in a class (relationship) can be characterised as limited in time or, vice versa, globalpossibly modelling legacy classes (relationships). For example, the company schema (Figure 1) models the membership of objects in the Employee class as time-invariant while objects in the Manager class have a limited lifespan as member of that class. Timestamping is the basis for associating the notion of lifecycle 37] to the object/relationship instances as members of a given class/relationship (more details are given in Section 6.1). Section 5 shows how evolution constraints can be formalised. 
Evolution Constraints. They control the mechanism that rules dynamic aspects, i.e., what are the permissible transitions from one state of the database to the next one [6, 7, 22, 38]. When applied to classes we talk about Object Migration, i.e., the evolution of an object from being member of a class to being member of another class [29]. For example, an object in the Employee class may migrate to become an object of the Manager class or an object of the AreaManager class can evolve into a TopManager class. When object migration combines with timestamping we talk about Status Classes. In this case we specify constraints on the membership of an object in a class by splitting it into periods according to a given classification criterion. For example, existence of a manager object in the Manager class can include periods where the object is an active member of the class (e.g., the manager is currently on payroll), periods where its membership is suspended (e.g., the manager is on temporary leave), and a period where its membership is disabled (e.g., the manager has left the company) [22]. The notion of status for classes allows also for a fine grained notion of lifecycle which can now depend on the membership to a particular status of a class.

Evolution-related knowledge may be conveyed also through relationships. Generation relationships [28] between objects of class A and objects of class B (possibly equal to A) describe the fact that objects in B are generated by objects in A. For example, in a company database, the splitting of a department translates into the fact that the original department generates two (or more) new departments. Clearly, if A and B are temporal classes, a generation relationship with source A and target B entails that the lifecycle of a B object cannot start before the lifecycle of the related A object. This particular temporal framework, where related objects do not coexist in time, is a form of, so called, across-time relationships [7, 38]. Section [6 shows how timestamping can be formalised.

In the conceptual modelling literature, different notion of 'time' have been considered. Notably, the most relevant distinction is between the so called valid time - which is the time when a property holds, i.e., it is true in the representation of the world - and transaction time - which records the history of database states rather than the world history, i.e., it is the time when a fact is current in the database and can be retrieved. In the following, we will consider both timestamping and evolution constructs as ranging over the valid time dimension.

\section{Modelling Requirements}

This Section briefly illustrates the requirements that are frequently advocated in the literature on temporal data models when dealing with temporal constraints [34, 38].

- Orthogonality. Temporal constructors should be specified separately and independently for classes, relationships, and attributes. Depending on application requirements, the temporal support must be decided by the designer.

- Upward Compatibility. This term denotes the capability of preserving the non-temporal semantics of conventional (legacy) conceptual schemas when embedded into temporal schemas. 
- Snapshot Reducibility. Snapshots of the database described by a temporal schema are the same as the database described by the same schema, where all temporal constructors are eliminated and the schema is interpreted atemporally. Indeed, this property specifies that we should be able to fully rebuild a temporal database by starting from the single snapshots.

These requirements are not so obvious when dealing with evolving objects. The formalisation carried out in this chapter provides a data model able to respect these requirements also in presence of evolving objects. In particular, orthogonality affects mainly timestamping [37] and our formalisation satisfies this principle by introducing temporal marks that could be used to specify the temporal behaviour of classes, relationships, and attributes in an independent way (see Section [5). Upward compatibility and snapshot reducibility [34] are strictly related. Considered together, they allow to preserve the meaning of atemporal constructors. In particular, the meaning of classical constructors must be preserved in such a way that a designer could either use them to model classical databases, or when used in a genuine temporal setting their meaning must be preserved at each instant of time. We enforce upward compatibility by using global timestamps over legacy constructors (see Section 5 ). Snapshot reducibility is hard to preserve when dealing with generation relationships where involved object may not coexist. We enforce snapshot reducibility by a particular treatment of relationship typing (see Section 6.3).

\section{A Formalisation of Temporal Data Models}

To give a formal foundation to temporal conceptual models we briefly describe here how to associate a textual syntax to a generic EER/UML modelling language. Having a textual syntax at hand will facilitate the association of a model-theoretic semantics. In the next sections we will take advantage of such a model-theoretic temporal semantics to formally describe the temporal constructs we are interested in.

We consider a temporal conceptual model over a finite alphabet, $\mathcal{L}$, partitioned into the sets: $\mathcal{C}$ (class symbols), $\mathcal{A}$ (attribute symbols), $\mathcal{R}$ (relationship symbols), $\mathcal{U}$ (role symbols), and $\mathcal{D}$ (domain symbols). We consider $n$-ary relationships where roles from the $\mathcal{U}$ alphabet are used to distinguish the different components of a relationship, i.e., an $n$-ary relationship, $R$, connecting the (not necessarily distinct) classes $C_{1}, \ldots, C_{n}$, is defined as $R=\left\langle U_{1}: C_{1}, \ldots, U_{n}: C_{n}\right\rangle$. Standard EER/UML constructs can also be textually defined, like Attributes for both classes and relationships (we use the notation $\operatorname{ATT}(C)=\left\langle A_{1}: D_{1}, \ldots, A_{h}: D_{h}\right\rangle$ to denote all the attributes of a class $C$, and similarly for attributes of relationships); Participation Constraints denoting the cardinality in the participation of a class into a relationship; Isa for both classes and relationships (denoted as $C_{1} \mathrm{ISA}_{2}$ or $R_{1} \mathrm{ISA} R_{2}$, respectively); Disjointness and Covering constraints over a class hierarchy. For a complete set of EER/UML constructs and their textual definition we refer to $[3,4,4,19]$. 
In Figure 1 we show our running example of an EER schema for a company database where classes and relationships are denoted by boxes and diamonds, respectively; directed arrows stand for isa; double arrows denote a covering constraint; a circled ' $d$ ' denotes a disjoint hierarchy; participation constraints are indicated with numbers in round brackets; timestamps are denoted with S (snapshot) and $\mathrm{T}$ (temporary).

The model-theoretic semantics that gives a foundation to temporal modelling languages adopts the so called snapsho 11 representation of abstract temporal databases and temporal conceptual models [20]. Following the snapshot paradigm, relations of a temporal database are interpreted by a mapping function depending on a specific point in time. The flow of time $\mathcal{T}=\left\langle\mathcal{T}_{p},<\right\rangle$, where $\mathcal{T}_{p}$ is a set of time points (or chronons) and $<$ is a binary precedence relation on $\mathcal{T}_{p}$, is assumed to be isomorphic to either $\langle\mathbb{Z},<\rangle$ or $\langle\mathbb{N},<\rangle$. Thus, a standard relational database can be regarded as the result of mapping a temporal database from a specific time point in $\mathcal{T}$ to an atemporal database, with the assumption that the interpretation of both constants and the domain are invariant over time.

Definition 1 (Temporal Schemas Semantics). Let $\Sigma$ be a temporal schema. $A$ temporal database state for the schema $\Sigma$ is a tuple $\mathcal{B}=\left(\mathcal{T}, \Delta^{\mathcal{B}} \cup \Delta_{D}^{\mathcal{B}},{ }^{\mathcal{B}(t)}\right)$, such that: $\Delta^{\mathcal{B}}$ is a nonempty set of abstract objects disjoint from $\Delta_{D}^{\mathcal{B}} ; \Delta_{D}^{\mathcal{B}}=$ $\bigcup_{D_{i} \in \mathcal{D}} \Delta_{D_{i}}^{\mathcal{B}}$ is the set of basic domain values used in the schema $\Sigma$; and ${ }^{\mathcal{B}(t)}$ is a function that for each $t \in \mathcal{T}$ maps:

- Every basic domain symbol $D_{i}$ into a set $D_{i}^{\mathcal{B}(t)}=\Delta_{D_{i}}^{\mathcal{B}}$.

- Every class $C$ to a set $C^{\mathcal{B}(t)} \subseteq \Delta^{\mathcal{B}}$.

- Every relationship $R$ to a set $R^{\mathcal{B}(t)}$ of $\mathcal{U}$-labeled tuples over $\Delta^{\mathcal{B}}$-i.e., let $R=\left\langle U_{1}: C_{1}, \ldots, U_{n}: C_{n}\right\rangle$ be an n-ary relationship connecting the classes $C_{1}, \ldots, C_{n}$, then, $\forall t \in \mathcal{T} . \forall r \in R^{\mathcal{B}(t)} \rightarrow\left(r=\left\langle U_{1}: o_{1}, \ldots, U_{n}: o_{n}\right\rangle \wedge \forall i \in\right.$ $\left.\{1, \ldots, n\} . o_{i} \in C_{i}^{\mathcal{B}(t)}\right)$. We adopt the convention: $\left\langle U_{1}: o_{1}, \ldots, U_{n}: o_{n}\right\rangle \equiv$ $\left\langle o_{1}, \ldots, o_{n}\right\rangle$, when $\mathcal{U}$-labels are clear from the context.

- Every attribute $A$ to a set $A^{\mathcal{B}(t)} \subseteq \Delta^{\mathcal{B}} \times \Delta_{D}^{\mathcal{B}}$, such that, for each $C \in \mathcal{C}$, if $\operatorname{ATT}(C)=\left\langle A_{1}: D_{1}, \ldots, A_{h}: D_{h}\right\rangle$, then, $\forall t \in \mathcal{T} \cdot \forall o \in C^{\mathcal{B}(t)} \rightarrow(\forall i \in$ $\left.\{1, \ldots, h\}, \forall a_{i} \cdot\left\langle o, a_{i}\right\rangle \in A_{i}^{\mathcal{B}(t)} \rightarrow a_{i} \in D_{i}^{\mathcal{B}(t)}\right)$.

$\mathcal{B}$ is said a legal temporal database state if it satisfies all of the constraints expressed in the schemd?

Given such a set-theoretic semantics we are able to rigorously define some relevant modelling notions such as satisfiability, subsumption and derivation of new constraints by means of logical implication.

Definition 2. Let $\Sigma$ be a schema, $C \in \mathcal{C}$ a class, and $R \in \mathcal{R}$ a relationship. The following modelling notions can be defined:

\footnotetext{
${ }^{1}$ The snapshot model represents the same class of temporal databases as the so called timestamp model 33, 34 which adds a temporal attribute to each relation 20].

${ }^{2}$ We don't report here the semantics for temporal constraints since they will be discussed in details in the next Sections. As for the semantics of participation constraints, isa, disjointness and covering constraints we refer to [4].
} 
1. $C(R)$ is satisfiable if there exists a legal temporal database state $\mathcal{B}$ for $\Sigma$ such that $C^{\mathcal{B}(t)} \neq \emptyset\left(R^{\mathcal{B}(t)} \neq \emptyset\right)$, for some $t \in \mathcal{T}$;

2. $\Sigma$ is satisfiable if there exists a legal temporal database state $\mathcal{B}$ for $\Sigma$ such that at least one class of $\Sigma$ is not empty ( $\mathcal{B}$ is also said a model for $\Sigma$ );

3. $C_{1}\left(R_{1}\right)$ is subsumed by $C_{2}\left(R_{2}\right)$ in $\Sigma$ if every legal temporal database state for $\Sigma$ is also a legal temporal database state for $C_{1}$ ISA $_{2}\left(R_{1}\right.$ ISA $\left.R_{2}\right)$;

4. A schema $\Sigma^{\prime}$ is logically implied by a schema $\Sigma$ over the same alphabet if every legal temporal database state for $\Sigma$ is also a legal temporal database state for $\Sigma^{\prime}$.

\section{$5 \quad$ Timestamping}

A temporal model supports timestamping if it is able to distinguish between snapshot constructors - i.e., constructors with a global lifespan associated to each of their instances - temporary constructors - i.e., each of their instances has a limited lifespan - or mixed constructors - i.e., their instances can have either a global or a temporary existence. In the following, a class, relationship or attribute is called temporal if it is either temporary or mixed. The two temporal marks, S (snapshot) and T (temporary), introduced at the conceptual level (see Figure 1), together with unmarked constructors capture the temporal distinction between snapshot, temporary and mixed constructors. Notice that, the temporal behaviour of an attribute can be either globally forced, or locally defined when associated to single classes. Since the local constraint is more general we assume that attributes are locally temporally constrained. At the end of this section we also introduce two notions strictly related to timestamping: that one of a (temporal) key, and a variant of participation constraints called lifespan participation constraints. We now proceed with the semantics of timestamping that can be defined as follows (not quantified variables are assumed to be universally quantified):

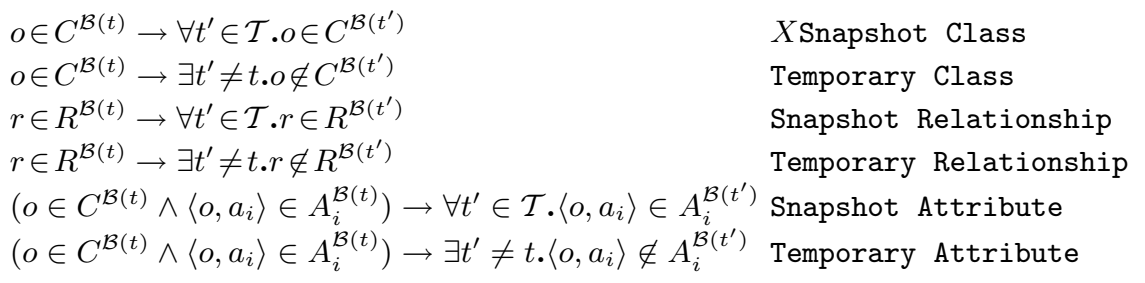

The following "classical" desirable features found in the literature of temporal conceptual modelling come as almost trivial logical implications form the above formalisation.

Proposition 1. (Timestamps: Logical Implications [4]) In every temporal schema supporting timestamping, the following temporal properties hold: 
1. Subclass of temporary classes are also temporary (similarly for relationships).

2. If exactly one of a whole set of snapshot subclasses partitioning ${ }^{3}$ a snapshot superclass is temporary, then, the whole set of classes is unsatisfiable.

3. Participants of snapshot relationships are either snapshot or unmarked classes.

4. Participants of snapshot relationships are snapshot when they participate at least once in the relationship.

5. A relationship is temporary if one of the participating classes is temporary.

On the other hand, nothing can be said about subclasses of snapshot or unmarked classes and classes participating to temporary or unmarked relationships: they can be snapshot, temporary, or unmarked classes. Since the domain of an attribute is not restricted to the classes they are attached to, the temporal behaviour of a class is independent of that of its attributes.

Example 1. Considering our running example of Figure 1, the following logical implications hold:

- Because Manager is a temporary class, then both AreaManager and TopManager are temporary classes; constraining either AreaManager or TopManager as snapshot classes would lead to a contradiction. On the other hand, even if Employee is a snapshot class, it is consistent to have Managera temporary class - as a subclass of Employee.

- Because OrganizationalUnit participates at least once in a snapshot relationship, then, it must be a snapshot class.

- Since InterestGroup participates in a partition of snapshot classes it must be a snapshot class, too.

- The fact that Manages must be a temporary relationship follows logically from our theory because the temporary class TopManager participates in the relationship.

- Since the temporal behaviour of classes is independent from that one of its attributes, the fact the Salary is a temporary attribute of the snapshot class Employee is admitted.

Key Constraints. As a byproduct of attribute timestamping we can define single-attribute keys (visualised in a schema as an underlined attribute, e.g., PaySlipNumber is a key for the class Employee) as a mandatory and singlevalued snapshot attribute that uniquely identifies objects of the class. Assuming that $A_{k e y}$ is a key for the class $C$, then the the following formalisation holds:

$$
\begin{array}{ll}
\left(o \in C^{\mathcal{B}(t)} \wedge\left\langle o, a_{\text {key }}\right\rangle \in A_{\text {key }}^{\mathcal{B}(t)}\right) \rightarrow \forall t^{\prime} \in \mathcal{T} \cdot\left\langle o, a_{k e y}\right\rangle \in A_{\text {key }}^{\mathcal{B}\left(t^{\prime}\right)} & \text { Snapshot Attribute } \\
o \in C^{\mathcal{B}(t)} \rightarrow \exists^{=1} a_{k e y} \in \Delta_{D}^{\mathcal{B}} \cdot\left\langle o, a_{k e y}\right\rangle \in A_{k e y}^{\mathcal{B}(t)} & \text { Mandatory \& } \\
a_{\text {key }} \in \Delta_{D}^{\mathcal{B}} \rightarrow \exists^{\leq 1} o \in C^{\mathcal{B}(t)} \cdot\left\langle o, a_{\text {key }}\right\rangle \in A_{k e y}^{\mathcal{B}(t)} & \text { Single-Valued } \\
\end{array}
$$

\footnotetext{
$\overline{3}$ The partition must be a disjoint covering.
} 


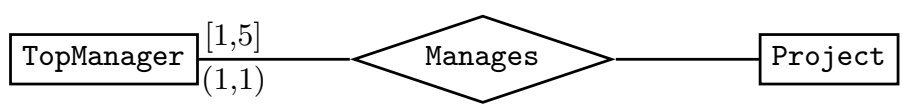

Fig. 2. Lifespan and "classical" participation constraints

Lifespan Participation Constraints. While classical participations constraints are evaluated at each point in time lifespan participation constraints (represented in a schema by a pair of values in square brackets) [26, 37, 39] are evaluated during the entire existence of the object. Notice that, since the set of instances of snapshot relationships does not change in time, there is no difference between "classical" and lifespan participation constraints for snapshot relationships. For example, if we want to state that a top manager should manage at most five different projects in his entire existence while still being constrained in managing exactly one project at a time, we can use a combination of the two participation constraints (see Figure 2). The model-theoretic semantics for lifespan participation is the following:

$$
\begin{aligned}
o \in C^{\mathcal{B}(t)} \rightarrow k \leq \# \bigcup_{t^{\prime} \in \mathcal{T}}\left\{r \in R^{\mathcal{B}\left(t^{\prime}\right)} \mid r[U]=o\right\} \leq m \text { Lifespan } & \text { Participation Constraint }
\end{aligned}
$$

\section{Evolution Constraints}

Evolution constraints contribute in modelling the temporal dynamic of an object. In this section we propose a formalisation of the basic temporal concepts that are at the root of advanced conceptual temporal models: status classes, distinguished in scheduled, active, suspended and disabled; transitions of objects between different classes; generation relationships asserting evolution constraints on objects linked by temporal relationships. In this section we aim at presenting a formal characterisation of the temporal conceptual modelling constructors capturing the evolution of objects.

\subsection{Status Classes}

The Status [7, 22, 37] is a conceptual notion associated to temporal classes to rule the lifecycle of their objects. It records the evolving state of membership of each object in the class. Following [37], status modelling includes up to four different statuses, and the allowed transitions between them:

- Scheduled. An object is scheduled if the planning of its existence within the class has to be recorded while its membership in the class will only become effective (active) some time later. For example, if a new project is approved but will not start until a later date the given project can be created as a new object in the Project class, with status scheduled for the valid time interval starting at the date of the approval decision and ending at the expected launching date. Each scheduled object will eventually become 
an active object. Supporting a scheduled status avoids the introduction of a new time type, the decision time [22].

- Active. The status of an object is active if the object is a full member of the class (and therefore conforms to its type). For example, a currently ongoing project is an active member, at time now, of the Project class.

- Suspended. This status qualifies objects that exist as members of the class, but are to be seen as temporarily inactive members of the class [22]. An employee taking a temporary leave of absence is an example of what can be considered as a suspended employee. Only active objects can be suspended. A suspended object was in the past an active one.

- Disabled. This status is used to specify that the object's membership in the class has expired. While logically deleted, disabled objects are kept for some specific application purposes, e.g., statistical analyses. A disabled object was in the past an active member of the class (an object cannot be created in the disabled status). It can never again become a non-disabled member of that class (e.g., an expired project cannot be reactivated).

Let $C$ be a temporal (i.e., temporary or mixed) class. We capture status transition of membership in $C$ by associating to $C$ the following status classes: Scheduled-C, Suspended-C, Disabled-C. In particular, status classes are constrained by the hierarchy of Figure 3 (where $C$ may also be mixed) that classifies $C$ instances according to their actual status. To preserve upward compatibility we do not explicitly introduce an active class, but assume by default that the name of the class itself denotes the set of active objects, i.e., Active-C $\equiv$ C. Note that, since membership of objects into snapshot classes is global, i.e., objects are always active, the notion of status classes does not apply to snapshot classes.

To capture the intended meaning of status classes, we define ad-hoc constraints and then show that such constraints capture the evolving behaviour of status classes as described in the literature 22, 37]. First of all, disjointness and isa constraints between statuses of a class $C$ can be described as illustrated in

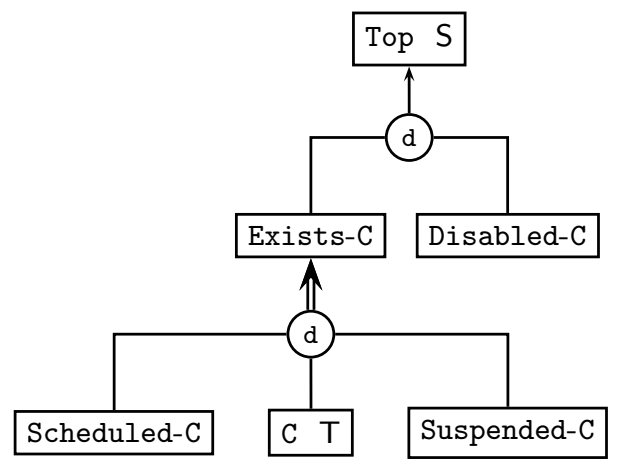

Fig. 3. Status classes 
Figure 3. where Top is supposed to be a snapshot class which represents the universe of abstract objects (i.e., Top ${ }^{\mathcal{B}(t)} \equiv \Delta^{\mathcal{B}}$ ). Other than hierarchical constraints, the intended semantics of status classes induces the following rules that are related to their temporal behaviour:

(EXISTS) Existence persists until Disabled.

$$
o \in \text { Exists-C }^{\mathcal{B}(t)} \rightarrow \forall t^{\prime}>t .\left(o \in \text { Exists-C } C^{\mathcal{B}\left(t^{\prime}\right)} \vee o \in \text { Disabled-C } C^{\mathcal{B}\left(t^{\prime}\right)}\right)
$$

(DISAB1) Disabled persists.

$$
o \in \text { Disabled-C } \mathrm{C}^{\mathcal{B}(t)} \rightarrow \forall t^{\prime}>t . o \in \text { Disabled-C } \mathrm{C}^{\mathcal{B}\left(t^{\prime}\right)}
$$

(DisAB2) Disabled was Active in the past. $o \in$ Disabled-C $\mathrm{C}^{\mathcal{B}(t)} \rightarrow \exists t^{\prime}<t . o \in \mathrm{C}^{\mathcal{B}\left(t^{\prime}\right)}$

(Susp) Suspended was Active in the past. $o \in$ Suspended-C $\mathrm{C}^{\mathcal{B}(t)} \rightarrow \exists t^{\prime}<t . o \in \mathrm{C}^{\mathcal{B}\left(t^{\prime}\right)}$

(SCH1) Scheduled will eventually become Active. $o \in$ Scheduled-C $\mathrm{C}^{\mathcal{B}(t)} \rightarrow \exists t^{\prime}>t . o \in \mathrm{C}^{\mathcal{B}\left(t^{\prime}\right)}$

(ScH2) Scheduled can never follow Active. $o \in \mathrm{C}^{\mathcal{B}(t)} \rightarrow \forall t^{\prime}>t . o \notin$ Scheduled-C $\mathrm{C}^{\mathcal{B}\left(t^{\prime}\right)}$

As a consequence of the above formalisation the following set of new rules can be derived.

Proposition 2 (Status Classes: Logical Implications [7]). Given a temporal schema supporting status classes, then, the following logical implications hold:

1. Disabled classes will never become active anymore.

2. The scheduled status persists until the class become active.

3. A scheduled class cannot evolve directly into a disabled status.

Temporal applications often use concepts that are derived from the notion of object statuses, e.g., the lifespan of a temporal object or its birth and death instants. Hereinafter we provide formal definitions for these concepts.

Lifespan and related notions. The lifespan of an object w.r.t. a class describes the temporal instants where the object can be considered a member of the class. With the introduction of status classes we can distinguish between the following

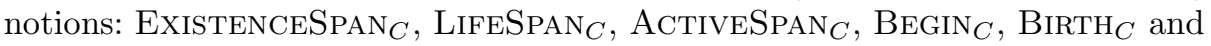
$\mathrm{DEATH}_{C}$. They are functions which depend on the object membership to the status classes associated to a temporal class $C$.

The existencespan of an object describes the temporal instants where the object is either a scheduled, active or suspended member of a given class. More formally, ExistenCESPAN $C: \Delta^{\mathcal{B}} \rightarrow 2^{\mathcal{T}}$, such that:

$$
\operatorname{ExistenCESPAN}_{C}(o)=\left\{t \in \mathcal{T} \mid o \in \operatorname{Exists}_{-} C^{\mathcal{B}(t)}\right\}
$$

The lifespan of an object describes the temporal instants where the object is an active or suspended member of a given class (thus, $\operatorname{LiFESPAN}_{C}(o) \subseteq$ $\left.\operatorname{ExistenceSpan}_{C}(o)\right)$. More formally, LifESPAN ${ }_{C}: \Delta^{\mathcal{B}} \rightarrow 2^{\mathcal{T}}$, such that: 


$$
\operatorname{LIFESPAN}_{C}(o)=\left\{t \in \mathcal{T} \mid o \in \mathrm{C}^{\mathcal{B}(t)} \cup \text { Suspended-C } \mathrm{C}^{\mathcal{B}(t)}\right\}
$$

The activespan of an object describes the temporal instants where the object is an active member of a given class (thus, $\operatorname{ACTiveSpan}_{C}(o) \subseteq \operatorname{LifESPAN}_{C}(o)$ ). More formally, ACTIVESPAN $C: \Delta^{\mathcal{B}} \rightarrow 2^{\mathcal{T}}$, such that:

$$
\operatorname{ACTiveSpan}_{C}(o)=\left\{t \in \mathcal{T} \mid o \in \mathrm{C}^{\mathcal{B}(t)}\right\}
$$

The functions Begin $C$ and Death ${ }_{C}$ associate to an object the first and the last appearance, respectively, of the object as a member of a given class, while BIRTH $_{C}$ denotes the first appearance as an active object of that class. More formally, BEGIN $C$, BiRTH $C$, DeATH $C: \Delta^{\mathcal{B}} \rightarrow \mathcal{T}$, such that:

$$
\begin{aligned}
& \operatorname{Begin}_{C}(o)=\min \left(\operatorname{ExistenceSpan}_{C}(o)\right) \\
& \operatorname{BiRTh}_{C}(o)=\min \left(\operatorname{ActiveSpan}_{C}(o)\right) \equiv \min \left(\operatorname{LifeSpan}_{C}(o)\right) \\
& \operatorname{DeAth}_{C}(o)=\max \left(\operatorname{LifeSpan}_{C}(o)\right)
\end{aligned}
$$

We could still speak of existencespan, lifespan or activespan for snapshot classes, but in this case they all collapse to the full time line, $\mathcal{T}$. Furthermore, $\operatorname{BEGIN}_{C}(o)$ $=\operatorname{BiRTh}_{C}(o)=-\infty$, and $\operatorname{DeATH}_{C}(o)=+\infty$ either when $C$ is a snapshot class or in cases of instances existing since ever and/or living forever.

\subsection{Transition}

Transition constraints [29, 37] have been introduced to model the phenomenon called object migration. A transition records objects migrating from a source class to a target class. At the schema level, it expresses that the instances of the source class may migrate into the target class. Two types of transitions have been considered: dynamic evolution, when objects cease to be instances of the source class to become instances of the target class, and dynamic extension, when the creation of the target instance does not force the removal of the source instance. For example, considering the company schema (Figure 1), if we want to record data about the promotion of area managers into top managers we can specify a dynamic evolution from the class AreaManager to the class TopManager. We can also record the fact that a mere employee becomes a manager by defining a dynamic extension from the class Employee to the class Manager (see Figure (4). Regarding the graphical representation, as illustrated in Figure 4, we use a dashed arrow pointing to the target class and labeled with either DEX or DEV denoting dynamic extension and evolution, respectively.

Specifying a transition between two classes means that: a) We want to keep track of such migration; $b$ ) Not necessarily all the objects in the source or in the target participate in the migration; c) When the source class is a temporal class, migration only involves active or suspended objects - thus, neither disabled nor scheduled objects can take part in a transition.

In the following, we present a formalisation that satisfies the above requirements. We represent transitions by introducing a new class denoted by either $\operatorname{DEX}_{C_{1}, C_{2}}$ or $\mathrm{DEV}_{C_{1}, C_{2}}$ for dynamic extension and evolution, respectively. More 


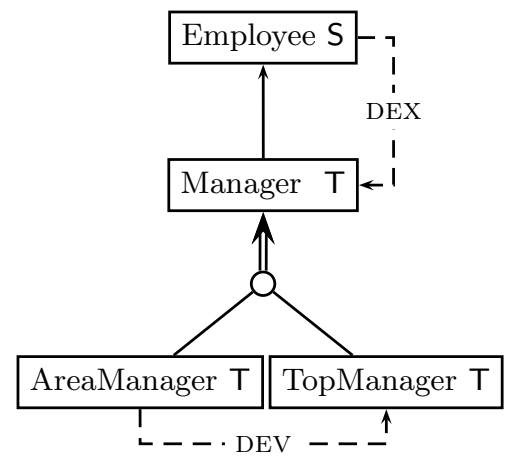

Fig. 4. Transitions employee-to-manager and area-to-top manager

formally, in case of a dynamic extension between classes $C_{1}, C_{2}$ the following semantic equation holds:

$$
o \in \operatorname{DEX}_{C_{1}, C_{2}}^{\mathcal{B}(t)} \rightarrow\left(o \in\left(\text { Suspended-C } \mathrm{C}_{1}{ }^{\mathcal{B}(t)} \cup \mathrm{C}_{1}{ }^{\mathcal{B}(t)}\right) \wedge o \notin \mathrm{C}_{2}{ }^{\mathcal{B}(t)} \wedge o \in C_{2}^{\mathcal{B}(t+1)}\right)
$$

In case of a dynamic evolution between classes $C_{1}, C_{2}$ the source object cannot remain active in the source class. Thus, the following semantic equation holds:

$$
\begin{aligned}
& o \in \operatorname{DEV}_{C_{1}, C_{2}}^{\mathcal{B}(t)} \rightarrow(o \in\left(\text { Suspended-C } \mathrm{C}_{1} \mathcal{B}(t) \cup \mathrm{C}_{1} \mathcal{B}(t)\right) \wedge o \notin \mathrm{C}_{2}{ }^{\mathcal{B}(t)} \wedge \\
&\left.o \in C_{2}^{\mathcal{B}(t+1)} \wedge o \notin C_{1}^{\mathcal{B}(t+1)}\right)
\end{aligned}
$$

Finally, we formalise the case where the source $\left(C_{1}\right)$ and/or the target $\left(C_{2}\right)$ totally participate in a dynamic extension/evolution (at schema level we add mandatory cardinality constraints on DEX/DEV links):

$$
\begin{array}{lll}
o \in C_{1}^{\mathcal{B}(t)} \rightarrow \exists t^{\prime}>t . o \in \operatorname{DEx}_{C_{1}, C_{2}}^{\mathcal{B}\left(t^{\prime}\right)} & \text { Source Total } & \text { Transition } \\
o \in C_{2}^{\mathcal{B}(t)} \rightarrow \exists t^{\prime}<t . o \in \operatorname{DEx}_{C_{1}, C_{2}}^{\mathcal{B}\left(t^{\prime}\right)} & \text { Target Total Transition } \\
o \in C_{1}^{\mathcal{B}(t)} \rightarrow \exists t^{\prime}>t . o \in \operatorname{DEV}_{C_{1}, C_{2}}^{\mathcal{B}\left(t^{\prime}\right)} & \text { Source Total Evolution } \\
o \in C_{2}^{\mathcal{B}(t)} \rightarrow \exists t^{\prime}<t . o \in \operatorname{DEV}_{C_{1}, C_{2}}^{\mathcal{B}\left(t^{\prime}\right)} & \text { Target } & \text { Total Evolution }
\end{array}
$$

An interesting set of consequences of the above proposed modelling of dynamic transitions are shown in the following proposition.

Proposition 3 (Transition: Logical Implications [7]). Given a schema supporting transitions for objects, then the following logical implications hold:

1. The classes $\mathrm{DEX}_{C_{1}, C_{2}}$ and $\mathrm{DEV}_{C_{1}, C_{2}}$ are temporary classes; actually, they hold at single time points.

2. Objects in the classes $\mathrm{DEX}_{C_{1}, C_{2}}$ and $\mathrm{DEV}_{C_{1}, C_{2}}$ cannot be disabled as $C_{2}$.

3. The target class $C_{2}$ cannot be snapshot (it becomes temporary in case of both Source Total Transition and Target Total Evolution constraints).

4. As a consequence of dynamic evolution, the source class, $C_{1}$, cannot be snapshot (and it becomes temporary in case of Source Total Evolution constraints). 
5. Dynamic evolution cannot be specified between a class and one of its sub-classes.

6. Dynamic extension between disjoint classes logically implies Dynamic evolution.

\subsection{Generation Relationships}

Generation relationships [7, 28, 36, 37] represent processes that lead to the emergence of new objects starting from a set of existing objects. In their most generic form, a generation relationship can have a collection of objects as source and a collection of objects as target. For example (see Figure 5), assuming an organisation remodels its departments, it may be that an existing department is split into two new departments, while two existing departments are merged into a single new department and three existing departments are reorganised as two new departments. Cardinality constraints can be added to specify the cardinality of sets involved in a generation. For example, if we want to record the fact that a group of managers proposes at most one new project at a time a generation relationship from Manager to Project can be defined with the cardinality "at most one" on the manager side.

Depending whether the source objects are preserved (as member of the source class) or disabled by the generation process, we distinguish between production and transformation relationships, respectively. Managers creating projects is an example of the former, while departmental reorganisation is an example of the latter. At the conceptual level we introduce two marks for generation relationships: GP for production and GT for transformation relationships, and an arrow pointing to the target class (see Figure 5).

We model generation as binary relationships connecting a source class to a target one, with the target being in its scheduled status: $\operatorname{REL}(R)=$ <source : $C_{1}$, target : Scheduled- $\left.\mathrm{C}_{2}\right\rangle$. The semantics of production relationships, $R$, is described by the following equation:

$$
\left\langle o_{1}, o_{2}\right\rangle \in R^{\mathcal{B}(t)} \rightarrow\left(o_{1} \in C_{1}^{\mathcal{B}(t)} \wedge o_{2} \in \text { Scheduled-C }{ }_{2}^{\mathcal{B}(t)} \wedge o_{2} \in C_{2}^{\mathcal{B}(t+1)}\right)
$$
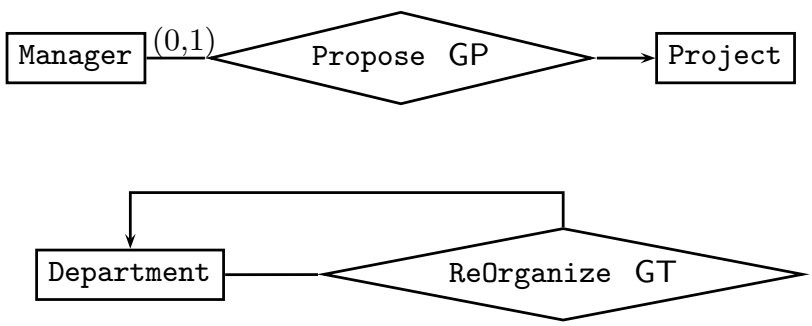

Fig. 5. Production and transformation generation relationships 
Thus, objects active in the source class produce objects active in the target class at the next point in time. A production relationship is a case of across-time relationships [7]-i.e., relationships connecting objects which are active in the connected classes at different points in time - where the use of status classes allows us to preserve snapshot reducibility. Indeed, for each pair of objects, $\left\langle o_{1}, o_{2}\right\rangle$, belonging to a generation relationships $o_{1}$ is active in the source while $O_{2}$ is scheduled in the target.

The case of transformation is captured by the following semantic equation:

$$
\begin{array}{r}
\left\langle o_{1}, o_{2}\right\rangle \in R^{\mathcal{B}(t)} \rightarrow\left(o_{1} \in C_{1}^{\mathcal{B}(t)} \wedge o_{1} \in \text { Disabled-C }_{1}{ }^{\mathcal{B}(t+1)} \wedge\right. \\
\left.o_{2} \in \text { Scheduled-C }_{2}{ }^{\mathcal{B}(t)} \wedge o_{2} \in C_{2}^{\mathcal{B}(t+1)}\right)
\end{array}
$$

Thus, objects active in the source generate objects active in the target at the next point in time while the source objects cease to exist as member of the source. As for production relationships, transformations are special cases of across-time relationships.

Proposition 4 (Generation: Logical Implications [7]). The following logical implications hold as a consequence of the generation semantics:

1. A generation relationship, $R$, is temporary; actually, it is instantaneous.

2. The target class, $C_{2}$, cannot be snapshot $\left(C_{2}\right.$ must be temporary if it participates at least once).

3. Objects participating as target cannot be disabled.

4. If $R$ is a transformation relationship, then, $C_{1}$ cannot be snapshot $\left(C_{1}\right.$ must be temporary if it participates at least once).

Note that the Department class that is both the source and target of a transformation relationship (Figure 5) can no longer be snapshot (as it was in Example 1) and must be changed to temporary. Furthermore, as a consequence of this new timestamp for the Department class, InterestGroup is now a genuine mixed class.

Starting with the next section we provide a correspondence between temporal conceptual schemas and theories expressed in temporal description logics.

\section{The Temporal Description Logic}

As the description logic $\mathcal{D} \mathcal{L R}$ has been used to reason over conceptual models 14 , 17, 18] in this chapter we use a temporal extension of $\mathcal{D} \mathcal{L} \mathcal{R}$ to capture temporal conceptual models. The temporal description logic $\mathcal{D} \mathcal{L} \mathcal{R}_{\mathcal{U S}}$ [5, 25] combines the propositional temporal logic with Since and Until and the (non-temporal) description logic $\mathcal{D} \mathcal{L} \mathcal{R}$ [13, 17]. $\mathcal{D} \mathcal{L} \mathcal{R}_{\mathcal{U S}}$ can be regarded as a rather expressive fragment of the first-order temporal logic $L^{\{\text {since, until\} }}$ (cf. [20, 30]).

The basic syntactical types of $\mathcal{D} \mathcal{L R}_{\mathcal{U S}}$ are concepts (unary predicates) and $n$-ary relations of arity $\geq 2$. Starting from a set of atomic concepts (denoted 


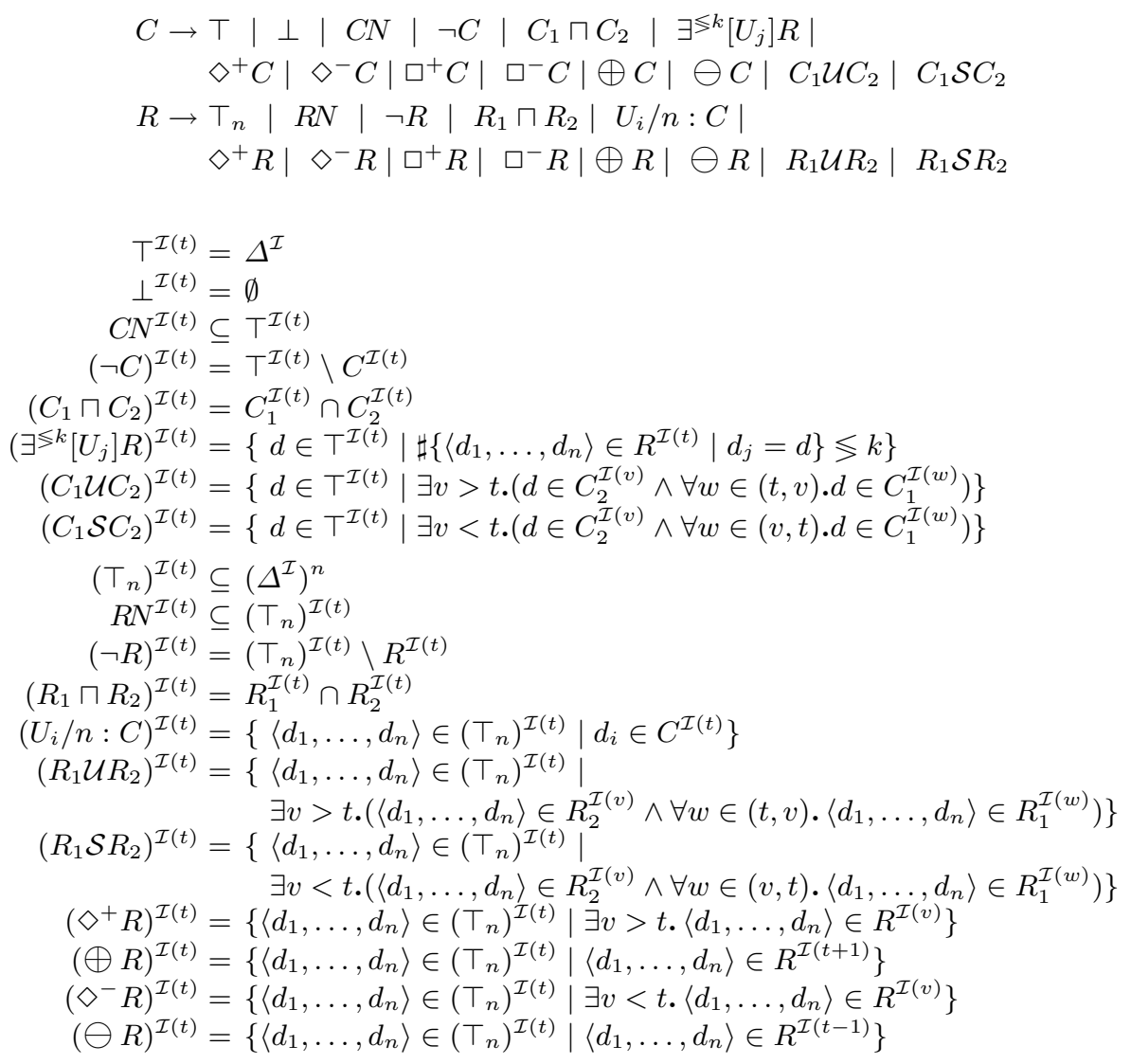

Fig. 6. Syntax and semantics of $\mathcal{D} \mathcal{L} \mathcal{R}_{\mathcal{U S}}$

by $C N$ ), a set of atomic relations (denoted by $R N$ ), and a set of role symbols (denoted by $U$ ) we hereinafter define inductively (complex) concepts and relation expressions as is shown in the upper part of Figure 6. where the binary constructors $(\sqcap, \sqcup, \mathcal{U}, \mathcal{S})$ are applied to relations of the same arity, $i, j, k, n$ are natural numbers, $i \leq n$, and $j$ does not exceed the arity of $R$.

The non-temporal fragment of $\mathcal{D} \mathcal{L} \mathcal{R}_{\mathcal{U S}}$ coincides with $\mathcal{D} \mathcal{L} \mathcal{R}$. For both concept and relation expressions all the Boolean constructors are available. The selection expression $U_{i} / n: C$ denotes an $n$-ary relation whose argument named $U_{i}$ $(i \leq n)$ is of type $C$; if it is clear from the context, we omit $n$ and write $\left(U_{i}: C\right)$. The projection expression $\exists{ }^{k}\left[U_{j}\right] R$ is a generalisation with cardinalities of the projection operator over the argument named $U_{j}$ of the relation $R$; the plain classical projection is $\exists \geq 1\left[U_{j}\right] R$ (we will use $\exists\left[U_{j}\right] R$ as a shortcut). It is also possible to use the pure argument position version of the language by replacing role symbols $U_{i}$ with the corresponding position numbers $i$. To show the expressive power of $\mathcal{D} \mathcal{L R}_{\mathcal{U S}}$ we refer to the next sections where $\mathcal{D} \mathcal{L} \mathcal{R}_{\mathcal{U S}}$ is used to capture various forms of temporal constraints. 
The model-theoretic semantics of $\mathcal{D} \mathcal{L} \mathcal{R}_{\mathcal{U S}}$ assumes a flow of time $\mathcal{T}=\left\langle\mathcal{T}_{p},<\right\rangle$, where $\mathcal{T}_{p}$ is a set of time points (or chronons) and $<$ a binary precedence relation on $\mathcal{T}_{p}$, is assumed to be isomorphic to $\langle\mathbb{Z},<\rangle$. The language of $\mathcal{D} \mathcal{L} \mathcal{R}_{\mathcal{U S}}$ is interpreted in temporal models over $\mathcal{T}$, which are triples of the form $\mathcal{I} \doteq\left\langle\mathcal{T}, \Delta^{\mathcal{I}},{ }^{\mathcal{I}(t)}\right\rangle$, where $\Delta^{\mathcal{I}}$ is non-empty set of objects (the domain of $\mathcal{I}$ ) and ${ }^{\mathcal{I}(t)}$ an interpretation function such that, for every $t \in \mathcal{T}$ (in the following the notation $t \in \mathcal{T}$ is used as a shortcut for $t \in \mathcal{T}_{p}$ ), every concepts $C$, and every $n$-ary relation $R$, we have $C^{\mathcal{I}(t)} \subseteq \Delta^{\mathcal{I}}$ and $R^{\mathcal{I}(t)} \subseteq\left(\Delta^{\mathcal{I}}\right)^{n}$. The semantics of concept and relation expressions is defined in the lower part of Figure [6 where $(u, v)=\{w \in \mathcal{T} \mid u<w<v\}$. For concepts, the temporal operators $\diamond^{+}$(some time in the future), $\oplus$ (at the next moment), and their past counterparts can be defined via $\mathcal{U}$ and $\mathcal{S}: \diamond^{+} C \equiv T \mathcal{U} C, \oplus C \equiv \perp \mathcal{U} C$, etc. The operators $\square^{+}$(always in the future) and $\square^{-}$(always in the past) are the duals of $\diamond^{+}$(some time in the future) and $\diamond^{-}$(some time in the past), respectively, i.e., $\square^{+} C \equiv \neg \diamond^{+} \neg C$ and $\square^{-} C \equiv \neg \diamond^{-} \neg C$, for both concepts and relations. The operators $\diamond^{*}$ (at some moment) and its dual $\square^{*}$ (at all moments) can be defined for both concepts and relations as $\diamond^{*} C \equiv C \sqcup \diamond^{+} C \sqcup \diamond^{-} C$ and $\square^{*} C \equiv C \sqcap \square^{+} C \sqcap \square^{-} C$, respectively.

A knowledge base, $\mathcal{K}$, is a finite set of $\mathcal{D} \mathcal{L} \mathcal{R}_{\mathcal{U S}}$ axioms of the form $C_{1} \sqsubseteq C_{2}$ and $R_{1} \sqsubseteq R_{2}$, with $R_{1}$ and $R_{2}$ being relations of the same arity. The notation $C_{1} \doteq C_{2}\left(R_{1} \doteq R_{2}\right)$ is a shortcut for $C_{1} \sqsubseteq C_{2}, C_{2} \sqsubseteq C_{1}\left(R_{1} \sqsubseteq R_{2}, R_{2} \sqsubseteq R_{1}\right)$. An interpretation $\mathcal{I}$ satisfies $C_{1} \sqsubseteq C_{2}\left(R_{1} \sqsubseteq R_{2}\right)$ if and only if the interpretation of $C_{1}\left(R_{1}\right)$ is included in the interpretation of $C_{2}\left(R_{2}\right)$ at all time, i.e., $C_{1}^{\mathcal{I}(t)} \subseteq C_{2}^{\mathcal{I}(t)}$ $\left(R_{1}^{\mathcal{I}(t)} \subseteq R_{2}^{\mathcal{I}(t)}\right)$, for all $t \in \mathcal{T}$. Various reasoning services can be defined in $\mathcal{D} \mathcal{L} \mathcal{R}_{\mathcal{U S}}$. A knowledge base, $\mathcal{K}$, is satisfiable if there is an interpretation that satisfies all the axioms in $\mathcal{K}$ (in symbols, $\mathcal{I} \models \mathcal{K}$ ). A knowledge base, $\mathcal{K}$, logically implies an axiom, $C_{1} \sqsubseteq C_{2}\left(R_{1} \sqsubseteq R_{2}\right)$, and write $\mathcal{K} \models C_{1} \sqsubseteq C_{2}\left(\Sigma \models R_{1} \sqsubseteq R_{2}\right)$, if we have $\mathcal{I} \models C_{1} \sqsubseteq C_{2}\left(\mathcal{I} \models R_{1} \sqsubseteq R_{2}\right)$ whenever $\mathcal{I} \models \mathcal{K}$. In this latter case, the concept $C_{1}$ (relation $R_{1}$ ) is said to be subsumed by the concepts $C_{2}$ (relation $R_{2}$ ) in the knowledge base $\mathcal{K}$. A concepts $C$ is satisfiable, given a knowledge base $\mathcal{K}$, if there exists a model $\mathcal{I}$ of $\mathcal{K}$ such that $C^{\mathcal{I}(t)} \neq \emptyset$ for some $t \in \mathcal{T}$, i.e., $\mathcal{K} \forall \models \subseteq \perp$. A relation $R$ is satisfiable, given a knowledge base $\mathcal{K}$, if there exists a model $\mathcal{I}$ of $\mathcal{K}$ such that $R^{\mathcal{I}(t)} \neq \emptyset$ for some $t \in \mathcal{T}$, i.e., $\mathcal{K} \not \models R \sqsubseteq \perp$. Finally, knowledge base satisfiability, concepts subsumption and relation satisfiability can be reduced to concepts satisfiability in the following way: $\mathcal{K} \forall \models 丁 \perp, \mathcal{K} \models C_{1} \sqcap \neg C_{2} \sqsubseteq \perp$, $\mathcal{K} \not \models \exists \geq 1\left[U_{j}\right] R \sqsubseteq \perp$ for some $j \leq n$ where $n$ is the arity of $R$, respectively.

While $\mathcal{D} \mathcal{L R}$ knowledge bases are fully able to capture atemporal EER/UML schemas [14, 17, 18] - i.e., given an EER schema there is an equi-satisfiable $\mathcal{D} \mathcal{L} \mathcal{R}$ knowledge base - in the following sections we use $\mathcal{D} \mathcal{L} \mathcal{R}_{\mathcal{U S}}$ knowledge bases to capture temporal EER/UML schemas with both timestamping and evolution constraints.

\section{Encoding Temporal Schemas in Description Logics}

We start by briefly summarising how knowledge bases in the description logic $\mathcal{D} \mathcal{L R}$ can capture conceptual schemas. The correspondence we report here is based on a mapping introduced by [14, 18, 19] for atemporal EER models. 
Informally, the encoding works as follows. Class and relationship symbols in a conceptual diagram are mapped into $\mathcal{D} \mathcal{L} \mathcal{R}$ concept names and relation names (with the same arity of the original relationship), respectively. Domain symbols are mapped into additional concept names, pairwise disjoint. Attributes of classes are mapped to binary relation names in $\mathcal{D} \mathcal{L} \mathcal{R}$ with number restrictions stating the cardinality of the attribute to distinguish between single- and multi-valued attributes. Isa links between classes or between relationships are mapped using $\mathcal{D} \mathcal{L} \mathcal{R}$ axioms. Generalised hierarchies with disjointness and covering constraints can be captured using Boolean connectives. Cardinality constraints are mapped using number restriction in $\mathcal{D} \mathcal{L} \mathcal{R}$.

Let us consider the class diagram depicted in Figure 1 and representing (a portion of) a company database. According to the diagram, all managers are employees and are partitioned into area managers and top managers. This information can be represented by means of the following $\mathcal{D} \mathcal{L} \mathcal{R}$ axioms:

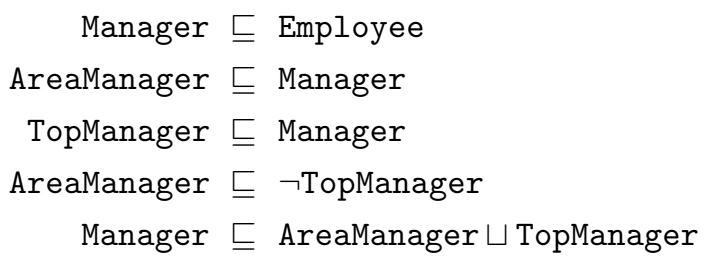

Binary relation names of the form $A \sqsubseteq$ From: $\top \sqcap$ To: $\top$ capture attributes. Each employee has three functional attributes (by default, we assume that attributes are single-valued and mandatory), Salary, PaySlipNumber, with integer values, and Name, with string values; here we show only the first:

$$
\text { Employee } \sqsubseteq \exists^{=1}\left[\text { from]Salary } \sqcap \exists^{=1}[\text { from] (Salary } \sqcap \text { to } / 2 \text { : Integer })\right.
$$

The binary relationship Works-for has Employees as domain, while the range is restricted to Projects:

$$
\text { Works-for } \sqsubseteq \text { emp/2: Employee } \sqcap \text { act } / 2 \text { : Project }
$$

Each top manager manages exactly one project, while a project must involve at least three employees:

$$
\begin{aligned}
\text { TopManager } \sqsubseteq \exists^{=1}[\mathrm{man}] \text { Manages } \\
\text { Project } \sqsubseteq \exists^{\geq 3}[\text { act] Works-For }
\end{aligned}
$$

Temporal properties expressed in the diagram are mapped using temporal operators in $\mathcal{D} \mathcal{L R}_{\mathcal{U S}}$. In the following we will show how to extend the above translation in order to capture both timestamping and evolution constraints.

\section{Encoding Timestamping}

Timestamping is the ability to distinguish between snapshot constructors-i.e., constructors with a global lifespan associated to each of their instances - temporary 
constructors - i.e., each of their instances has a limited lifespan-or mixed constructors - i.e., their instances can have either a global or a temporary existence. Timestamps for both classes and relationships are captured by the following $\mathcal{D} \mathcal{L R}_{\mathcal{U}}$ axioms (remember that $\square^{*}$ is the "at all time" operator while $\diamond^{*}$ is the "at some time" operator, see Section 7):

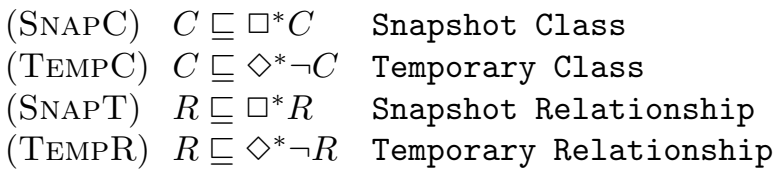

Considering timestamping for attributes we first recall that attributes are captured in $\mathcal{D} \mathcal{L} \mathcal{R}$ as binary relations. Thus, the following $\mathcal{D} \mathcal{L R}_{\mathcal{U S}}$ axioms hold:

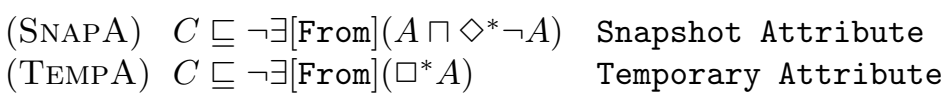

Key Constraints. We now show the $\mathcal{D} \mathcal{L R}_{\mathcal{U S}}$ axioms that capture the notion of single-attribute keys (see the case of a pay slip number that uniquely identifies an employee in Figure 1). We need three axioms: the first to specify that a key is a snapshot attribute, the second to characterise a key as mandatory and singlevalued, and the last axiom to specify uniqueness. Assuming that $A_{k e y}$ is a key for the class $C$, then its semantics is captured by the following $\mathcal{D} \mathcal{L} \mathcal{R}_{\mathcal{U S}}$ axioms:

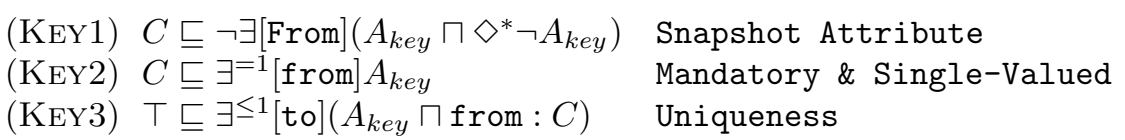

Lifespan Participation Constraints. Lifespan participation constraints (see Figure 2) are formalised in $\mathcal{D} \mathcal{L R}_{\mathcal{U S}}$ using a combination of number restrictions and temporal operators for relations:

\section{(LPC) $C \sqsubseteq \exists^{\geq \mathrm{k}}[\mathrm{U}] \diamond^{*} \mathrm{R} \sqcap \exists^{\leq \mathrm{m}}[\mathrm{U}] \diamond^{*} \mathrm{R}$ Lifespan Participation Constraint}

The standard logical implications due to timestamping and showed in Proposition 1 can be rephrased in terms of $\mathcal{D} \mathcal{L} \mathcal{R}_{\mathcal{U S}}$ logical implications.

Proposition 5 (Timestamps: Logical Implications [4])). In every temporal schema supporting timestamping, the following temporal properties hold:

1. Subclass of temporary classes are also temporary (similarly for relationships). $\left\{C_{1} \sqsubseteq C, C \sqsubseteq \diamond^{*} \neg C\right\} \models C_{1} \sqsubseteq \diamond^{*} \neg C_{1}$

2. If exactly one of a whole set of snapshot subclasses partitioning a snapshot superclass is temporary, then, the whole set of classes is unsatisfiable (we consider a three class partition).

$\left\{C_{0} \doteq C_{1} \sqcup C_{2}, C_{1} \sqsubseteq \neg C_{2}, C_{0} \sqsubseteq \square^{*} C_{0}, C_{1} \sqsubseteq \square^{*} C_{1}, C_{2} \sqsubseteq \diamond^{*} \neg C_{2}\right\} \models C_{i} \sqsubseteq$ $\perp, \quad i=0,1,2$

${ }^{4}$ The axioms consider a local temporal behaviour for attributes. To associate a global behaviour to an attribute we consider it as a binary relationship and apply the axioms for timestamping relationships. 
3. Participants of snapshot relationships are either snapshot or unmarked classes. $\left\{R \sqsubseteq \square^{*} R, R \sqsubseteq U_{i}: C_{i}, C_{i} \sqsubseteq \diamond^{*} \neg C_{i}\right\} \models R \sqsubseteq \perp$

4. Participants of snapshot relationships are snapshot when they participate at least once in the relationship.

$\left\{R \sqsubseteq \square^{*} R, R \sqsubseteq U_{i}: C_{i}, C_{i} \sqsubseteq \exists\left[U_{i}\right] R\right\} \models C_{i} \sqsubseteq \square^{*} C_{i}$

5. A relationship is temporary if one of the participating classes is temporary. $\left\{R \sqsubseteq U_{i}: C_{i}, C_{i} \sqsubseteq \diamond^{*} \neg C_{i}\right\} \models R \sqsubseteq \diamond^{*} \neg R$

\section{Encoding Status Classes}

Status classes record the evolving state of membership of each object in the class. We distinguish four status: scheduled, active, suspended and disabled. $\mathcal{D} \mathcal{L} \mathcal{R}_{\mathcal{U S}}$ axioms are able to fully capture the hierarchical constraints of Figure 3 , Moreover, the semantic equations formalising status classes are captured by the following set of $\mathcal{D} \mathcal{L} \mathcal{R}_{\mathcal{U S}}$ axioms:

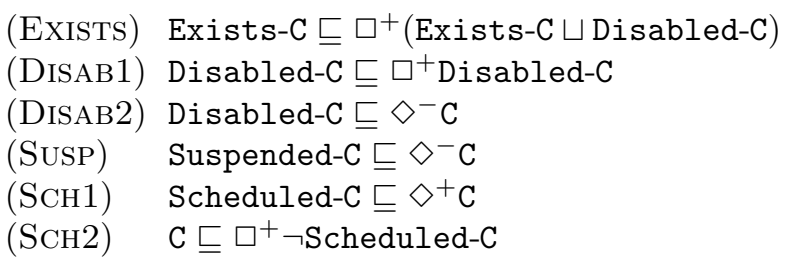

We denote with $\Sigma_{s t}$ the above set of axioms together with the $\mathcal{D} \mathcal{L} \mathcal{R}_{\mathcal{U S}}$ axioms that capture the hierarchy of Figure 3. We can now rephrase the logical implications involving status classes showed in Proposition 2 as $\mathcal{D} \mathcal{L} \mathcal{R}_{\mathcal{U S}}$ logical implications.

Proposition 6 (Status Classes: Logical Implications [7]). Given the set of $\mathcal{D} \mathcal{L R}_{\mathcal{U S}}$ axioms $\Sigma_{\text {st }}$ that capture status classes, the following logical implications hold:

1. Disabled will never become active anymore.

$\Sigma_{s t} \models$ Disabled-C $\sqsubseteq \square^{+} \neg \mathrm{C}$

2. Scheduled persists until active.

$\Sigma_{s t} \models$ Scheduled-C $\sqsubseteq$ Scheduled-CUC

3. Scheduled cannot evolve directly to Disabled.

$\Sigma_{s t} \models$ Scheduled-C $\sqsubseteq \oplus \neg$ Disabled-C

\section{Encoding Transition}

Transition constraints model the so called object migration. They are distinguished in dynamic evolution - when objects cease to be instances of the source class to become instances of the target class - and dynamic extension - when the creation of the target instance does not force the removal of the source instance. We represent transitions by introducing a new class denoted by either $\operatorname{DEX}_{C_{1}, C_{2}}$ or $\mathrm{DEV}_{C_{1}, C_{2}}$ for dynamic extension and evolution, respectively. The $\mathcal{D} \mathcal{L} \mathcal{R}_{\mathcal{U S}}$ axioms capturing these temporal constraints are: 
(DEX) $\quad \operatorname{DEX}_{C_{1}, C_{2}} \sqsubseteq$ (Suspended-C $\left.\mathrm{C}_{1} \sqcup \mathrm{C}_{1}\right) \sqcap \neg \mathrm{C}_{2} \sqcap \oplus C_{2}$

(DEV) $\operatorname{DEV}_{C_{1}, C_{2}} \sqsubseteq$ (Suspended-C $\left.\mathrm{C}_{1} \sqcup \mathrm{C}_{1}\right) \sqcap \neg \mathrm{C}_{2} \sqcap \oplus\left(C_{2} \sqcap \neg C_{1}\right)$

The $\mathcal{D} \mathcal{L}_{\mathcal{U S}}$ axioms capturing the cases where the source $\left(C_{1}\right)$ and/or the target $\left(C_{2}\right)$ totally participate in a dynamic extension/evolution are:
(STT) $C_{1} \sqsubseteq \diamond^{+} \operatorname{DEX}_{C_{1}, C_{2}}$
Source Total Transition
(TTT) $C_{2} \sqsubseteq \diamond^{-} \operatorname{DEX}_{C_{1}, C_{2}}$
Target Total Transition
(STE) $C_{1} \sqsubseteq \diamond^{+} \mathrm{DEV}_{C_{1}, C_{2}}$
Source Total Evolution
(TTE) $C_{2} \sqsubseteq \diamond^{-} \mathrm{DEV}_{C_{1}, C_{2}}$
Target Total Evolution

We can now rephrase the logical implications involving transition constraints showed in Proposition 3 as $\mathcal{D} \mathcal{L} \mathcal{R}_{\mathcal{U S}}$ logical implications.

Proposition 7 (Transition: Logical Implications [7]). Let $\Sigma_{t r}=\{(\mathrm{DEV})$, $(D e x)\}$, then the following logical implications hold:

1. The classes $\mathrm{DEX}_{C_{1}, C_{2}}$ and $\mathrm{DEV}_{C_{1}, C_{2}}$ are temporary classes; actually, they hold at single time points.

$\Sigma_{s t} \cup \Sigma_{t r} \models \operatorname{DEX}_{C_{1}, C_{2}} \sqsubseteq \bigoplus \neg \operatorname{DEX}_{C_{1}, C_{2}} \sqcap \ominus \neg \operatorname{DEX}_{C_{1}, C_{2}}$

$\Sigma_{s t} \cup \Sigma_{t r} \models \operatorname{DEV}_{C_{1}, C_{2}} \sqsubseteq \bigoplus \neg \mathrm{DEV}_{C_{1}, C_{2}} \sqcap \ominus \neg \mathrm{DEV}_{C_{1}, C_{2}}$

2. Objects in the classes $\mathrm{DEX}_{C_{1}, C_{2}}$ and $\mathrm{DEV}_{C_{1}, C_{2}}$ cannot be disabled as $C_{2}$. $\Sigma_{s t} \cup \Sigma_{t r} \models \operatorname{DEX}_{C_{1}, C_{2}} \sqsubseteq \neg$ Disabled-C 2

$\Sigma_{s t} \cup \Sigma_{t r} \models \operatorname{DEV}_{C_{1}, C_{2}} \sqsubseteq \neg$ Disabled-C $C_{2}$

3. The target class $C_{2}$ cannot be snapshot (it becomes temporary in case of both (TTT) and (TTE) constraints).

$\Sigma_{s t} \cup \Sigma_{t r} \models \operatorname{DEX}_{C_{1}, C_{2}} \sqsubseteq \diamond^{*}\left[C_{2} \sqcap\left(\diamond^{+} \neg C_{2} \sqcup \diamond^{-} \neg C_{2}\right)\right]$

4. As a consequence of dynamic evolution, the source class, $C_{1}$, cannot be snapshot (and it becomes temporary in case of (STE) constraints).

$\Sigma_{s t} \cup \Sigma_{t r} \models \operatorname{DEV}_{C_{1}, C_{2}} \sqsubseteq \diamond^{*}\left[C_{1} \sqcap\left(\diamond^{+} \neg C_{1} \sqcup \diamond^{-} \neg C_{1}\right)\right]$

5. Dynamic evolution cannot be specified between a class and one of its subclasses.

$\Sigma_{s t} \cup \Sigma_{t r} \cup\left\{C_{2} \sqsubseteq C_{1}\right\}=\operatorname{DEV}_{C_{1}, C_{2}} \sqsubseteq \perp$

6. Dynamic extension between disjoint classes logically implies Dynamic evolution.

$\Sigma_{s t} \cup \Sigma_{t r} \cup\left\{C_{1} \sqsubseteq \neg C_{2}\right\} \models \operatorname{DEX}_{C_{1}, C_{2}} \sqsubseteq \mathrm{DEV}_{C_{1}, C_{2}}$

\section{Encoding Generation Relationships}

Generation relationships lead to the emergence of new objects starting from a set of existing objects. Depending whether the source objects are preserved (as member of the source class) or disabled, we distinguish between production and transformation relationships, respectively. The $\mathcal{D} \mathcal{L} \mathcal{R}_{\mathcal{U S}}$ axioms capturing the production and transformation semantics are:

(PROD) $R \sqsubseteq$ source : $C_{1} \sqcap$ target : (Scheduled- $\left.C_{2} \sqcap \oplus C_{2}\right)$

(Trans) $R \sqsubseteq$ source: $\left(C_{1} \sqcap \oplus\right.$ Disabled- $\left.C_{1}\right) \sqcap$ target: (Scheduled- $\left.\mathrm{C}_{2} \sqcap \oplus C_{2}\right)$

We can now rephrase the logical implications involving generation relationships showed in Proposition 4 as $\mathcal{D} \mathcal{L} \mathcal{R}_{\mathcal{U}}$ logical implications. 
Proposition 8 (Generation: Logical Implications [7]). The following logical implications hold as a consequence of the $\mathcal{D} \mathcal{L R}_{\mathcal{U S}}$ axioms capturing generation relationships:

1. A generation relationship, $R$, is temporary; actually, it is instantaneous. $\Sigma_{\text {st }} \cup\{(\mathrm{PROD})\} \models R \sqsubseteq \square^{+} \neg R \sqcap \square^{-} \neg R$

2. The target class, $C_{2}$, cannot be snapshot $\left(C_{2}\right.$ must be temporary if it participates at least once).

$\Sigma_{s t} \cup\{(\mathrm{PROD})\} \models R \sqsubseteq$ target $: \diamond^{*}\left[C_{2} \sqcap\left(\diamond^{+} \neg C_{2} \sqcup \diamond^{-} \neg C_{2}\right)\right]$

3. Objects participating as target cannot be disabled. $\Sigma_{\text {st }} \cup\{(\mathrm{PROD})\} \models R \sqsubseteq$ target $: \neg$ Disabled $-\mathrm{C}_{2}$

4. If $R$ is a transformation relationship, then, $C_{1}$ cannot be snapshot $\left(C_{1}\right.$ must be temporary if it participates at least once).

$\Sigma_{s t} \cup\{($ Trans $)\} \models R \sqsubseteq$ source $: \diamond^{*}\left[C_{1} \sqcap\left(\diamond^{+} \neg C_{1} \sqcup \diamond^{-} \neg C_{1}\right)\right]$

\subsection{Correctness of the Encoding}

To prove that reasoning on temporal schemas can be done by reasoning on their $\mathcal{D} \mathcal{L R}_{\mathcal{U S}}$ translation, we need to prove the correctness of the encoding. That temporal schemas with timestamping and transition constraints can be encoded as description logic theories has been proven correct in [4, 5] by establishing a precise correspondence between legal database states of temporal schemas and models of the corresponding description logic theories. This result can be easily extended to the full set of temporal constraints presented here.

Theorem 1 (Correctness of the encoding). Let $\Sigma$ be a temporal schema. Then, $\Sigma$ admits a legal database state if and only if the corresponding $\mathcal{D} \mathcal{L} \mathcal{R}_{\mathcal{U}}$ knowledge base encoding the schema has a model.

This characterisation allows us to support the reasoning on temporal conceptual models, as in Definition 2, by using the reasoning services of $\mathcal{D} \mathcal{L} \mathcal{R}_{\mathcal{U S}}$. On the other hand, since reasoning with $\mathcal{D} \mathcal{L} \mathcal{R}_{\mathcal{U S}}$ theories is undecidable, in the following section we present interesting scenarios where reasoning become decidable together with their respective complexity results.

\section{Complexity of Reasoning on Temporal Models}

As this chapter shows, the temporal description logic $\mathcal{D} \mathcal{L} \mathcal{R}_{\mathcal{U S}}$ is able to fully capture temporal schemas with both timestamping and evolution constraints. On the other hand, reasoning over $\mathcal{D} \mathcal{L} \mathcal{R}_{\mathcal{U S}}$ knowledge bases, i.e., checking satisfiability, subsumption and logical implications, turns out to be undecidable [5]. The main reason for this is the possibility to couple the evolution of concepts with the possibility to postulate that a binary relation does not vary in time (i.e., global relations). Note that, showing that temporal schemas can be mapped into $\mathcal{D} \mathcal{L R}_{\mathcal{U S}}$ axioms does not necessarily imply that reasoning over temporal schemas is an undecidable problem. Unfortunately, [1] shows that the undecidable Halting 
Problem can be encoded as the problem of class satisfiability w.r.t. a temporal schema with, among the others, the following constructs: disjoint and covering constraints, sub-relationships, timestamping on both classes and relationships, and evolution constraints.

On the other hand, the fragment, $\mathcal{D} \mathcal{L} \mathcal{R}_{\mathcal{U}}^{-}$, of $\mathcal{D} \mathcal{L} \mathcal{R}_{\mathcal{U S}}$ deprived of the ability to talk about temporal persistence of $n$-ary relations, for $n \geq 2$, is decidable. Indeed, reasoning in $\mathcal{D} \mathcal{L R}_{\mathcal{U}}^{-}$is an EXPTIME-complete problem [5]. This result gives us an useful scenario where reasoning over temporal schemas becomes decidable. In particular, if we forbid timestamping for relationships (i.e., relationships are just unmarked and interpreted as mixed constructors) reasoning on temporal models with just class timestamping but full evolution constraints can be reduced to reasoning over $\mathcal{D} \mathcal{L} \mathcal{R}_{\mathcal{U}}^{-}$. The problem of reasoning in this setting is complete for EXPTIME since the EXPTIME-complete problem of reasoning with $\mathcal{A L C}$ knowledge bases can be captured by such schemas [14].

We maintain decidability also by allowing full timestamping (i.e., timestamping for relationships, attributes and classes) but dropping evolution constraints. This is the basic temporal conceptual modelling scenario where temporal marks allow to distinguish between temporary and global constructs (this scenario also allows for both temporal keys and lifespan participation constraints). This scenario is decidable since it is possible to encode temporal schemas without evolution constraints by using a combination between the description logic $\mathcal{D} \mathcal{L} \mathcal{R}$ and the epistemic modal logic S5 (see [12] for the exact mapping). Reasoning over $\mathcal{D} \mathcal{L} \mathcal{R}_{S 5}$ has been proved to be decidable and 2-EXPTIME-complete [11] by extending a previous result on the logic $\mathcal{A L C}_{S 5}[25]$.

Other interesting scenarios currently under investigation are the cases where the temporal expressivity is maintained in its full capability (i.e., both full timestamping and evolution constraints) but some of the constructs used at the conceptual level are dropped. In particular, by dropping isa between relationships and/or partitioning constraints we could regain decidability in the full temporal scenario. In this case we can use description logics from the DL-Lite family [2, 15, 16] to capture these weaker forms of conceptual schemas (see [8] for an exhaustive description of the data models that can be captured inside $D L$ Lite). A demoralisation of $D L$-Lite has been proposed in [10] where reasoning is showed to be EXPSPACE-complete. As a future work we plan to study the mapping of the various temporal constructs presented here in the temporal extension of $D L$-Lite and to investigate a tight complexity bound for the resulting temporal data modelling language.

\section{Conclusions}

This chapter summarises the various proposals appeared in the literature about temporal conceptual data models within a formal framework. We presented a model-theoretic semantics for different temporal constructs grouped along two generic categories, i.e., timestamping and evolution constraints. The given formal semantics clarifies the meaning of the modelling constructors and also gives 
a rigorous definition to relevant design support tasks such as satisfiability of schemas, classes and relationships; subsumption for both classes and relationships; general logical implication. Furthermore, for each constructor we have shown how desirable properties can be derived as logical implications from the proposed formalisation.

We have been able to show how temporal schemas can be equivalently expressed using a subset of first-order temporal logic, i.e., $\mathcal{D} \mathcal{L} \mathcal{R}_{\mathcal{U S}}$, the description logic $\mathcal{D} \mathcal{L} \mathcal{R}$ extended with the temporal operators Since and Until. While $\mathcal{D} \mathcal{L} \mathcal{R}_{\mathcal{U S}}$ is an undecidable language, several decidable sub-languages can be used to reason over temporal schemas. Since these sub-languages usually do not mix timestamping with evolution constraints, we are currently investigating new scenarios where, by weakening the atemporal expressiveness of the conceptual model, we regain decidability of the full temporal setting. We started to work on these encouraging scenarios by using $D L$-Lite as the atemporal DL and extending it to capture time varying domains.

\section{Acknowledgements}

We would like to thank our colleagues Roman Kontchakov, Carsten Lutz, Federica Mandreoli, Christine Parent, Vladislav Ryzhikov, Stefano Spaccapietra, David Toman, Frank Wolter, and Michael Zakharyaschev with whom we carried out most of the work presented in this chapter.

\section{References}

[1] Artale, A.: Reasoning on temporal conceptual schemas with dynamic constraints. In: 11th Int. Symposium on Temporal Representation and Reasoning (TIME 2004). IEEE Computer Society, Los Alamitos (2004); also in Proc. of the 2004 Int. Workshop on Description Logics (DL 2004)

[2] Artale, A., Calvanese, D., Kontchakov, R., Zakharyaschev, M.: DL-Lite in the light of first-order logic. In: Proc. of AAAI 2007, pp. 361-366 (2007)

[3] Artale, A., Franconi, E.: Temporal ER modeling with description logics. In: Akoka, J., Bouzeghoub, M., Comyn-Wattiau, I., Métais, E. (eds.) ER 1999. LNCS, vol. 1728, pp. 81-95. Springer, Heidelberg (1999)

[4] Artale, A., Franconi, E., Mandreoli, F.: Description logics for modelling dynamic information. In: Chomicki, J., van der Meyden, R., Saake, G. (eds.) Logics for Emerging Applications of Databases. LNCS, Springer, Heidelberg (2003)

[5] Artale, A., Franconi, E., Wolter, F., Zakharyaschev, M.: A temporal description logic for reasoning over conceptual schemas and queries. In: Flesca, S., Greco, S., Leone, N., Ianni, G. (eds.) JELIA 2002. LNCS(LNAI), vol. 2424, pp. 98-110. Springer, Heidelberg (2002)

[6] Artale, A., Parent, C., Spaccapietra, S.: Modeling the evolution of objects in temporal information systems. In: Dix, J., Hegner, S.J. (eds.) FoIKS 2006. LNCS, vol. 3861, pp. 22-42. Springer, Heidelberg (2006)

[7] Artale, A., Parent, C., Spaccapietra, S.: Evolving objects in temporal information systems. Annals of Mathematics and Artificial Intelligence 50(1-2), 5-38 (2007) 
[8] Artale, A., Calvanese, D., Kontchakov, R., Ryzhikov, V., Zakharyaschev, M.: Reasoning over extended ER models. In: Parent, C., Schewe, K.-D., Storey, V.C., Thalheim, B. (eds.) ER 2007. LNCS, vol. 4801, pp. 277-292. Springer, Heidelberg (2007)

[9] Artale, A., Cesarini, F., Soda, G.: Describing database objects in a concept language environment. IEEE Trans. on Knowledge and Data Engineering 8(2), 345$351(1996)$

[10] Artale, A., Kontchakov, R., Lutz, C., Wolter, F., Zakharyaschev, M.: Temporalising tractable description logics. In: 14th International Symposium on Temporal Representation and Reasoning (TIME 2007). IEEE Computer Society Press, Los Alamitos (2007)

[11] Artale, A., Lutz, C., Toman, D.: A description logic of change. In: Int. Joint Conference on Artificial Intelligence (IJCAI 2007), Hyderabad, India (January 2007)

[12] Artale, A., Toman, D.: Decidable reasoning over timestamped conceptual models. In: Proc. of the 21st Int. Workshop on Description Logics (DL 2008), Dresden, Germany (May 2008)

[13] Baader, F., Calvanese, D., McGuinness, D., Nardi, D., Patel-Schneider, P.F. (eds.): Description Logic Handbook: Theory, Implementation and Applications. Cambridge University Press, Cambridge (2002)

[14] Berardi, D., Calvanese, D., De Giacomo, G.: Reasoning on UML class diagrams. Artificial Intelligence 168(1-2), 70-118 (2005)

[15] Calvanese, D., De Giacomo, G., Lembo, D., Lenzerini, M., Rosati, R.: DL-Lite: Tractable description logics for ontologies. In: Proc. of the 20th Nat. Conf. on Artificial Intelligence (AAAI 2005), pp. 602-607 (2005)

[16] Calvanese, D., De Giacomo, G., Lembo, D., Lenzerini, M., Rosati, R.: Data complexity of query answering in description logics. In: Proc. of the 10th Int. Conf. on the Principles of Knowledge Representation and Reasoning (KR 2006), pp. 260-270 (2006)

[17] Calvanese, D., De Giacomo, G., Lenzerini, M.: On the decidability of query containment under constraints. In: Proc. of the 17th ACM SIGACT SIGMOD SIGART Sym. on Principles of Database Systems (PODS 1998), pp. 149-158 (1998)

[18] Calvanese, D., Lenzerini, M., Nardi, D.: Description logics for conceptual data modeling. In: Chomicki, J., Saake, G. (eds.) Logics for Databases and Information Systems. Kluwer, Dordrecht (1998)

[19] Calvanese, D., Lenzerini, M., Nardi, D.: Unifying class-based representation formalisms. J. of Artificial Intelligence Research 11, 199-240 (1999)

[20] Chomicki, J., Toman, D.: Temporal logic in information systems. In: Chomicki, J., Saake, G. (eds.) Logics for Databases and Information Systems, ch. 1. Kluwer, Dordrecht (1998)

[21] Combi, C., Degani, S., Jensen, C.S.: Capturing temporal constraints in temporal ER models. In: Li, Q., Spaccapietra, S., Yu, E., Olivé, A. (eds.) ER 2008. LNCS, vol. 5231. Springer, Heidelberg (2008)

[22] Etzion, O., Gal, A., Segev, A.: Extended update functionality in temporal databases. In: Etzion, O., Jajodia, S., Sripada, S. (eds.) Dagstuhl Seminar 1997. LNCS, vol. 1399, pp. 56-95. Springer, Heidelberg (1998)

[23] Finger, M., McBrien, P.: Temporal conceptual-level databases. In: Gabbay, D., Reynolds, M., Finger, M. (eds.) Temporal Logics - Mathematical Foundations and Computational Aspects, pp. 409-435. Oxford University Press, Oxford (2000) 
[24] Franconi, E., Sattler, U.: A data warehouse conceptual data model for multidimensional aggregation. In: Proc. of the Workshop on Design and Management of Data Warehouses (DMDW 1999) (1999)

[25] Gabbay, D., Kurucz, A., Wolter, F., Zakharyaschev, M.: Many-dimensional modal logics: theory and applications. Studies in Logic. Elsevier, Amsterdam (2003)

[26] Gregersen, H., Jensen, J.S.: Conceptual modeling of time-varying information. Technical Report TimeCenter TR-35, Aalborg University, Denmark (1998)

[27] Gregersen, H., Jensen, J.S.: Temporal Entity-Relationship models - a survey. IEEE Transactions on Knowledge and Data Engineering 11(3), 464-497 (1999)

[28] Gupta, R., Hall, G.: An abstraction mechanism for modeling generation. In: Proc. of ICDE 1992, pp. 650-658 (1992)

[29] Hall, G., Gupta, R.: Modeling transition. In: Proc. of ICDE 1991, pp. 540-549 (1991)

[30] Hodkinson, I., Wolter, F., Zakharyaschev, M.: Decidable fragments of first-order temporal logics. Annals of Pure and Applied Logic 106, 85-134 (2000)

[31] Horrocks, I., Patel-Schneider, P.F., van Harmelen, F.: From SHIQ and RDF to OWL: The making of a web ontology language. Journal of Web Semantics 1(1), 7-26 (2003)

[32] Jensen, C.S., Clifford, J., Gadia, S.K., Hayes, P., Jajodia, S., et al.: The Consensus Glossary of Temporal Database Concepts. In: Etzion, O., Jajodia, S., Sripada, S. (eds.) Temporal Databases - Research and Practice, pp. 367-405. Springer, Heidelberg (1998)

[33] Jensen, C.S., Snodgrass, R.T.: Temporal data management. IEEE Transactions on Knowledge and Data Engineering 111(1), 36-44 (1999)

[34] Jensen, C.S., Soo, M., Snodgrass, R.T.: Unifying temporal data models via a conceptual model. Information Systems 9(7), 513-547 (1994)

[35] McBrien, P., Seltveit, A.H., Wangler, B.: An Entity-Relationship model extended to describe historical information. In: Proc. of CISMOD 1992, Bangalore, India, pp. 244-260 (1992)

[36] Parent, C., Spaccapietra, S., Zimanyi, E.: The MurMur project: Modeling and querying multi-representation spatio-temporal databases. Information Systems 31(8), 733-769 (2006)

[37] Spaccapietra, S., Parent, C., Zimanyi, E.: Modeling time from a conceptual perspective. In: Int. Conf. on Information and Knowledge Management (CIKM 1998) (1998)

[38] Spaccapietra, S., Parent, C., Zimanyi, E.: Conceptual Modeling for Traditional and Spatio-Temporal Applications - The MADS Approach. Springer, Heidelberg (2006)

[39] Tauzovich, B.: Towards temporal extensions to the entity-relationship model. In: Proc. of the Int. Conf. on Conceptual Modeling (ER 1991). Springer, Heidelberg (1991)

[40] Theodoulidis, C., Loucopoulos, P., Wangler, B.: A conceptual modelling formalism for temporal database applications. Information Systems 16(3), 401-416 (1991) 